\title{
Radiological evaluation of the enhancement patterns of thickened gallbladder wall to differentiate benign from malignancy
}

\author{
Manavjit S Sandhu ${ }^{1}$, Yoganand Kommisetti ${ }^{1}$, Naveen Kalra ${ }^{1 *}$, Anmol Bhatia ${ }^{2}$, Rajesh Gupta ${ }^{3}$, \\ Ritambhara Nada ${ }^{4}$ and Niranjan Khandelwal ${ }^{1}$ \\ ${ }^{1}$ Department of Radiodiagnosis and Imaging, Post Graduate Institute of Medical Education and Research,Chandigarh, India. \\ ${ }^{2}$ Department of Gastroenterology, Post Graduate Institute of Medical Education and Research, Chandigarh, India. \\ ${ }^{3}$ Department of General surgery, Post Graduate Institute of Medical Education and Research, Chandigarh, India. \\ ${ }^{4}$ Department of Histopathology, Post Graduate Institute of Medical Education and Research, Chandigarh, India.
}

Received: November 8, 2016; Accepted: December 28, 2016; Published: January 9, 2017

*Corresponding author: Naveen Kalra, Department of Radio diagnosis and Imaging, Post Graduate Institute of Medical Education and Research, Chandigarh, India, Tel: 00917087009388; E mail: navkal2004@yahoo.com

\begin{abstract}
Objective: To evaluate the enhancement patterns of thickened gallbladder wall on MDCT and vascularity of gallbladder wall on Doppler Ultrasound, and to correlate imaging findings with pathological diagnosis in malignant and non-malignant conditions.

Methods: In this institute's ethics committee approved prospective study, 15 patients with gallbladder wall thickening ( $>3 \mathrm{~mm}$ on ultrasonography) underwent contrast enhanced twophase MDCT of abdomen and Doppler examination for gallbladder The gallbladder wall attenuation value on CT was measured by drawing Region of Interest and compared to liver parenchymal attenuation. Enhancement patterns of gallbladder wall on CT were analysed. The findings were correlated with pathological diagnosis.

Results: The presence of layered pattern of enhancement of gallbladder wall was seen more in carcinoma (85.7\%). Lack of layered pattern correlated with benign nature. The difference was statistically significant $(p=0.005)$. The finding of hyper-attenuation of gallbladder wall to that of liver parenchyma on arterial phase showed sensitivity, specificity, PPV, NPV values of $85.7 \%, 62.5 \%, 66 \%$ and $83.3 \%$ respectively for malignancy, while iso or hypo-attenuation of the wall showed sensitivity, specificity, PPV, NPV of $62.5 \%, 85.7 \%$, $83.3 \%$ and $66.7 \%$ respectively for chronic cholecystitis. Gallbladder wall intramural color flow was seen in eight patients, of which 4 had carcinoma and 4 chronic cholecystitis (difference was not statistically significant, $p=0.78$ ).

Conclusion: Analysis of gallbladder wall enhancement patterns along with objective measurement of gallbladder wall attenuation in a two-phase MDCT study of the upper abdomen may add to the radiologist's confidence in pre-operative differentiation of gallbladder carcinoma from benign etiologies.
\end{abstract}

Key words: Gall bladder; Thickening; Benign; Malignancy; CT

\section{Introduction}

Gallbladder cancer is the most common malignancy of the biliary tract and is the $5^{\text {th }}$ most common malignancy of gastrointestinal tract. Carcinoma of the gallbladder is more commonly seen in women (two to three times) than in men and its incidence steadily increases with age [1]. Thickening of gallbladder wall is a commonly detected finding on different imaging modalities. The finding itself is however non-specific and can result from multiple pathologic conditions including surgical and non-surgical disorders. The least common presentation of gallbladder carcinoma is focal or diffuse thickening of the gallbladder wall, and is difficult to diagnose, particularly in the early stages. Considering the non-specific nature of the clinical manifestations, which are indistinguishable from chronic cholecystitis, it is important to have an imaging tool for early detection of gallbladder cancer, because the management and prognosis of gallbladder carcinoma differs from benign etiologies. It is also well recognized that early detection, although a mere coincidental rarity, improves survival [2-4]. On the other hand, misinterpretation of diffuse gallbladder wall thickening in patients who do not have primarily gallbladder disease can lead to unnecessary cholecystectomy. This situation calls for an improved imaging strategy to differentiate between benign and malignant causes of thickened gall bladder wall in order to select patients for aggressive therapeutic efforts [4].

Computed tomography to differentiate cholecystitis from carcinoma has been evaluated as early as in 1984 [5], however the signs reported to be useful included detecting a focal mass, biliary obstruction at porta hepatis, invasion of liver and nodal metastases. Yun et al [6] evaluated different patterns of enhancement thickened gallbladder wall in arterial and venous phases and found them helpful in differentiating gallbladder carcinoma from chronic cholecystitis when using two-phase helical CT. The authors opined that two-phase spiral CT was more useful than single phase CT for differentiating between these two disease entities. Another retrospective study by Kim et al [7] aimed to differentiate the gallbladder 
cancer presenting with diffuse wall thickening from acute and chronic cholecystitis using analysis of the gallbladder wall enhancement patterns on MDCT portal venous phase images. The sensitivity for detecting the flat wall-thickening type of gallbladder cancer using the five-pattern analysis was found to be $82.8 \%$ and $75.9 \%$ and the positive predictive value was $80 \%$ and $84.6 \%$, respectively, for the two different observers. The present study was conducted to analyse the enhancement patterns of thickened gallbladder wall on MDCT, evaluate the vascularity pattern of the gallbladder wall on Doppler Ultrasound, and to correlate these pre-operative imaging findings with the histopathological/cytological examination in malignant and nonmalignant conditions with thickening of gallbladder wall.

\section{Materials and methods}

This prospective study was approved by the ethics committee of the institute. Fifteen consecutive patients in the age range of $20-90 y r s$ with a gallbladder wall thickness of more than $3 \mathrm{~mm}$ were included over a period of 12 months.

1. Exclusion criteA mass replacing the gallbladder.

2. Gall bladder mass with hepatic infiltration or adjacent organ infiltration.

3. Patients with ascites, in whom a clinical correlation suggests a non gall bladder disease that could cause the wall thickening, namely generalized edematous states of congestive heart failure, renal failure, cirrhosis, hypoalbuminemia, and patients with secondary inflammation of gallbladder as in acute pancreatitis.

\section{Pregnant women}

Informed written consent was taken from all the patients. Details of clinical history, examination and sonographic findings were recorded.

All the patients underwent a color Doppler and power Doppler examination to assess the vascularity of the gallbladder wall after a period of at least $4 \mathrm{hrs}$ of fasting. The power Doppler examination was done on a Philips HD11 scanner using 3-12 MHz high frequency linear and 2-5 MHz convex array probes and the findings were analysed for the presence of:

1. Layered pattern of gallbladder wall thickening.

2. Vascularity within the wall.

\section{Vascularity within the focal area of thickening.}

Presence of vascularity within the thickened gallbladder wall or focal areas of gallbladder wall thickening were tentatively considered as indicative of malignant gallbladder wall thickening.

These results were compared with that of the histopathological or cytological examination reports and the sensitivity and specificity of the various identified enhancement patterns and the vascularity on color/power Doppler to differentiate between benign and malignant causes of gallbladder wall thickening was determined.

This was followed by a contrast enhanced biphasic computed tomography scan on a multidetector CT scanner (Sensation 16, 16 detector slice scanner, Siemens medical solutions; or Toshiba 64 detector slice scanner, Toshiba) using intravenous non-ionic iodinated contrast material. Two phase CT scans were obtained in arterial and venous phases, at 35 and 70 seconds after the initiation of contrast material injection respectively.

The information obtained in the CT scans was analysed in the following manner:

1. Does the thickened gallbladder wall show a layered pattern?

2. The attenuation values of gallbladder wall and that of the liver parenchyma were measured in both arterial and portal venous phases. The measurements were done by manually drawing regions of interest (ROI) using a dedicated workstation. The gallbladder wall ROI area ranged from $0.07 \mathrm{~cm}^{2}$ to $0.15 \mathrm{~cm}^{2}$. While drawing the ROI in the liver parenchyma, care was taken to select an area which was devoid of large vessels or bile ducts, which could interfere with the attenuation measurements. The liver parenchyma ROI area ranged from $1.00 \mathrm{~cm}^{2}$ to $1.50 \mathrm{~cm}^{2}$.

3. Where there was a layered pattern, the thickness and attenuation values of each layer were measured separately.

4. The measured attenuation values of the gallbladder wall were compared to that of the liver parenchyma. They were categorised as hyper or hypo attenuating to that of liver parenchyma in case of attenuation difference of more than $10 \mathrm{HU}$. If the attenuation value was within $10 \mathrm{HU}$ of the liver parenchyma, it was categorised as iso attenuating to the liver parenchyma.

5. Based on these values the enhancement patterns were grouped as:

A. Type 1: Heterogenously hyper-enhancing thickened gallbladder wall without a layered pattern with persistent portal venous phase hyper-enhancement.

B. Type 2: Hyper-enhancing inner layer in arterial phase showing persistent portal venous phase enhancement.

C. Type 3: Hyper-enhancing inner layer in arterial phase showing iso or hypo-attenuation in portal venous phase.

D. Type 4: Iso/hypo attenuating gallbladder wall to that of liver parenchyma on arterial phase showing enhancement in portal venous phase.

E. Type 5: Iso/hypo attenuating gallbladder wall in arterial and in portal venous phase.

Amongst these patterns, types 1,2 , and 3 were considered as indicative of malignancy and the other patterns considered 
as benignancy. These patterns were compared with the histopathological or cytological examination reports for specificity and sensitivity of this analysis in differentiating malignant from non-malignant causes of gallbladder wall thickening.

\section{Statistical Analysis}

Statistical analysis was performed using SPSS 17.0 package.

1. The Chi square test was used to determine the significance of qualitative data between the gallbladder

Carcinoma and the cholecystitis groups.

2. An unpaired t-test was used to compare normally distributed continuous variables.

3. Sensitivity, specificity, and positive and negative predictive values were calculated for each variable.

4. Statistically significant differences were defined as those with $\mathrm{p}$ value $<0.05$.

\section{Results}

The age range of the patients was 45 to 83 years of age (mean 59.27 years). There were seven male and eight female patients. Out of the seven male patients five had cholecystitis, and two had malignancy. Among the females, five had malignancy and three had cholecystitis.

\section{Computed tomography (CT)}

\section{Wall thickness}

The gallbladder wall thickening measured on CT ranged from $4.5 \mathrm{~mm}$ to $20.9 \mathrm{~mm}$. The mean wall thickness in gallbladder carcinoma patients was $14.31 \mathrm{~mm}$. The mean wall thickness in cholecystitis patients was $10.57 \mathrm{~mm}$. The receiver operating characteristic (ROC) curve was constructed for increased gallbladder wall thickness as an indicator of malignancy. The area under the curve was 0.750 indicating that probability of carcinoma was higher if wall thickness was higher.

\section{Layered pattern}

The presence of layered pattern of gallbladder wall enhancement was observed in a total of 7 cases, 6 of which were finally diagnosed as carcinoma. Among those cases not showing a layered pattern, $88 \%$ (7 out of 8) finally turned out to be chronic cholecystitis. Pearson's Chi-square test showed chi square value of 8.040 with a p value of 0.005 .

\section{Focal or diffuse pattern of gallbladder wall thickening}

Eight cases showed diffuse gallbladder wall thickening, out of which five turned out to be benign and three turned out to be malignant. Out of the seven cases of focal gallbladder wall thickening, four turned out to be malignant and three turned out to be benign. No statistically significant association was seen between focal gallbladder wall thickening and its malignant nature (Chi-square value of 0.579 and $\mathrm{p}=0.44$ ).

\section{Measurement of attenuation values}

The attenuation value of the wall or its inner layer in arterial phase in malignant group ranged from 69 to $121 \mathrm{HU}$ with a mean value of $94.86 \mathrm{HU}$. The attenuation in arterial phase in cholecystitis group ranged from 38 to $101 \mathrm{HU}$ with a mean value of $64.5 \mathrm{HU}$. The difference in attenuation values was statistically significant $(\mathrm{p}<0.05)$.

The attenuation of inner wall on portal venous phase ranged from 52 to $101 \mathrm{HU}$ in the cholecystitis group and ranged from 72 to $116 \mathrm{HU}$ in the carcinoma group. The mean attenuation value in portal venous phase was 76.13 in cholecystitis group and 99.71 in gallbladder carcinoma group. However, the portal venous phase attenuation difference was not statistically significant.

The area under ROC curve for arterial phase wall attenuation was 0.849 and area under curve for portal venous phase wall attenuation was 0.857 . These results suggest that the chances of gallbladder carcinoma increase with increase in gallbladder wall attenuation value in both arterial and portal venous phases. ROC curves show sensitivity of $\sim 71 \%$ with specificity of $\sim 87 \%$ for an arbitrary cut off value of $97 \mathrm{HU}$ in portal venous phase and $94 \mathrm{HU}$ in arterial phase.

Six out of seven cases of gallbladder carcinoma (86\%) showed hyper-attenuation of the gallbladder wall or its inner layer in comparison to the liver parenchyma in the arterial phase. Conversely the finding of hyper-attenuation of gallbladder wall corresponded to malignancy in $66 \%$ of cases ( 6 out of 9). The remaining $33 \%$ of cases turned out to be benign.

Five out of eight cases $(62.5 \%)$ of cholecystitis showed iso or hypo-attenuation of the gallbladder wall or its inner layer in comparison to liver parenchyma in the arterial phase. Conversely the finding of iso or hypo-attenuation of the gallbladder wall corresponded to benignancy in $83.3 \%$ of cases ( 5 out of 6 ).

Based on the patterns of gallbladder wall enhancement, the benign pattern was observed in a total of six patients, five of whom had a final diagnosis of cholecystitis [Figure 1] and one case was false positive. The malignant pattern was observed in a total of 9 cases, out of which 6 had final diagnosis of carcinoma [Figure 2] and 3 cases were false positive [Figure 3-4]. Type1, type 2 and type 3 patterns combined yielded a positive predictive value of $66 \%$ with sensitivity of $86 \%$ for malignancy. For evaluation of benign aetiology, the pattern types 4 and 5 had a sensitivity of $62.5 \%$ and PPV of $83.3 \%$.

\section{Power Doppler}

On power Doppler ultrasonography, the visualisation of vascularity within the gallbladder wall was evaluated. A total of eight patients showed evidence of color flow within the thickened gallbladder wall. Out of these eight patients, four had a final diagnosis of carcinoma and four had the final diagnosis of cholecystitis. Seven patients did not show any evidence of color flow within the gallbladder wall. Four of these cases turned out to be cholecystitis and the other three turned out to be carcinoma. There was no statistically significant difference (Chi-square value of 0.07 , p value 0.78 ).

\section{Final diagnosis}

All the patients underwent either ultrasound guided fine 
needle aspiration cytology or surgery followed by histopathology for confirmation of the imaging diagnosis. Five patients underwent both FNAC and histopathological examination.

The final diagnosis was benignancy (cholecystitis) in a total of eight patients. Six of these cases underwent cholecystectomy and histopathology of the resected specimens confirmed the diagnosis of cholecystitis. Four of these eight patients underwent fine needle aspiration cytology under ultrasound guidance. Two of these four had subsequent cholecystectomy as mentioned above. One patient (case 6 in images section) opted out of surgery and was followed up on imaging, which showed contraction of the gallbladder 7 months later. One patient opted for surgery at a later date.

The final diagnosis was malignancy in a total of seven patients. Three underwent both FNAC and surgery followed by histopathology confirmation. One patient underwent surgery and histopathology confirmation without FNAC. The remaining three patients had FNAC evidence of malignancy and opted out of surgery. One of these patients developed subsequent biliary obstruction and ascites, and underwent palliative percutaneous

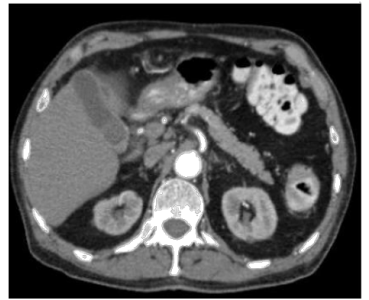

Figure1A

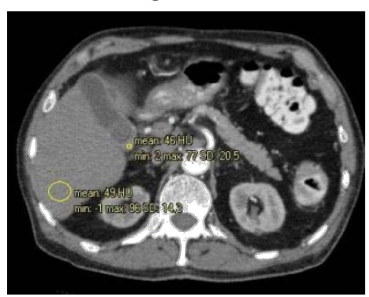

Figure 1c

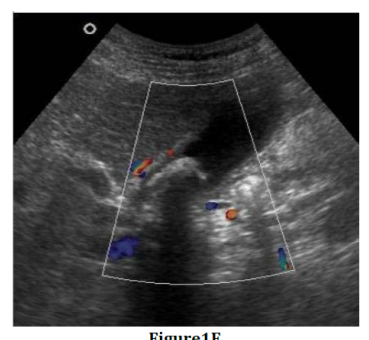

Figure1E

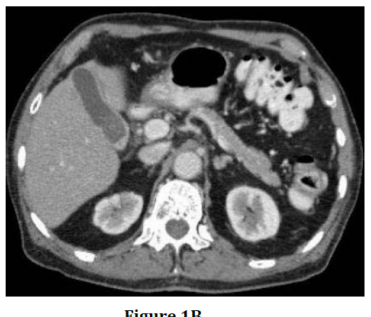

Figure 1B

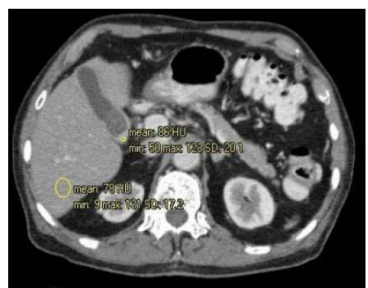

Figure 1D

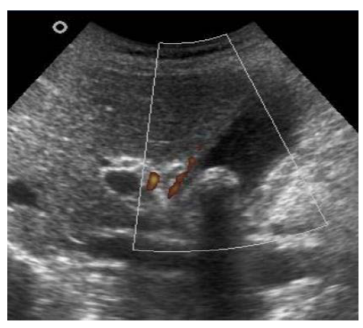

Figure1F
Figure 1: 64-year-old male with diffuse thickening of the gallbladder wall. Arterial phase images (A and C) show iso-attenuation of thickened gallbladder wall compared to the liver parenchyma. Portal venous phase images (B and D) show iso-attenuation compared to liver parenchyma. The pattern of enhancement was classified as type 5 (suggesting benignancy). Histopathology confirmed cholecystitis. Color Doppler (E) and power Doppler (F) images show color flow within the gallbladder wall.

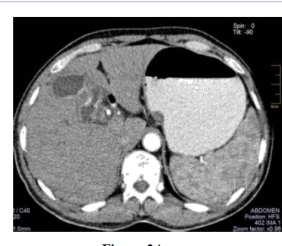

Figure 2.

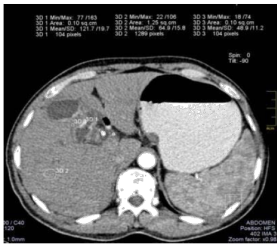

Figure 2C

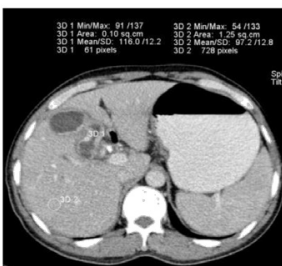

Figure 2

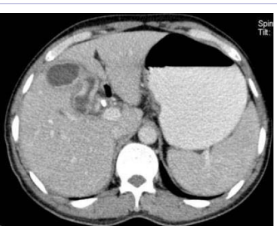

Figure 2B

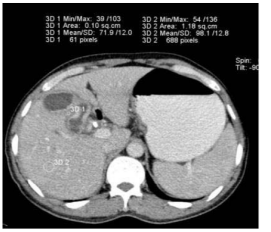

Figure 2D

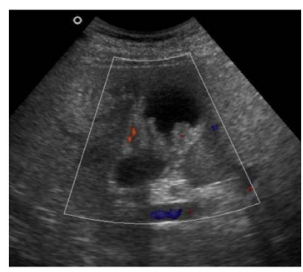

Figure 21

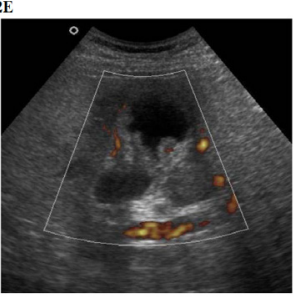

Figure $2 \mathrm{G}$

Figure 2: 55-year-old male with diffuse asymmetric thickening of the gallbladder wall. The arterial phase images (A and C) show hyper-attenuation of inner layer and hypo-attenuation of outer layer compared to liver parenchyma. Portal venous phase also shows inner layer hyperattenuation and outer layer hypo-attenuation compared to liver (B, D and E) Type of enhancement was classified as type 2 (suggesting malignancy). FNAC confirmed malignancy. Color Doppler (F) and power Doppler (G) images show color flow in the thickened wall in body.

trans-hepatic biliary drainage.

\section{Discussion}

Gallbladder wall thickening due to secondary causes can be differentiated based on clinical and imaging grounds, the preoperative differentiation between gallbladder malignancy and chronic cholecystitis is however difficult. Previous studies $[5,6]$ have attempted to differentiate between chronic cholecystitis and gallbladder carcinoma presenting as wall thickening on imaging. In the study done by Yun et al [6] to differentiate chronic cholecystitis from gallbladder carcinoma on two phase spiral CT, the mean wall thickness in gallbladder carcinoma was $12.6 \mathrm{~mm}$ and that of chronic cholecystitis was $6.9 \mathrm{~mm}$ with the difference being statistically significant $(\mathrm{p}<0.05)$. In our study the mean thicknesses of the wall in gallbladder malignancy group and cholecystitis group were $14.31 \mathrm{~mm}$ and $10.91 \mathrm{~mm}$ respectively. A cut off value of $13.45 \mathrm{~mm}$ for malignancy yielded sensitivity of $\sim 85 \%$ and a specificity of $\sim 75 \%$. There was significant statistical 
difference in the variances (Levene's test for equality of Variances showing $\mathrm{p}=0.023$ ). The independent sample t test showed a $\mathrm{p}$ value of 0.13 . Although malignancy becomes more likely, more studies on larger number of patients are needed to define a cutoff for this parameter.

The presence of a layered pattern of gallbladder wall on ultrasonography (striated appearance) has been associated with edematous non-malignant states [8, 9]. On computed tomography, some studies $[5,10]$ showed that the finding of disruption of the normal layers in a thickened gallbladder wall indicates malignancy. However, studies by Yun et al [6] and Kim et al [7] showed that gallbladder carcinoma can present with a layered pattern of enhancement on CT. In our study, 7 out of 15 cases $(46 \%)$ showed a layered pattern of enhancement on CT.

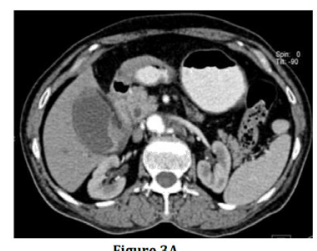

Figure 34

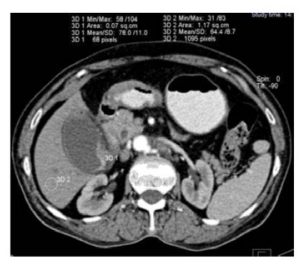

Figure $3 \mathrm{C}$

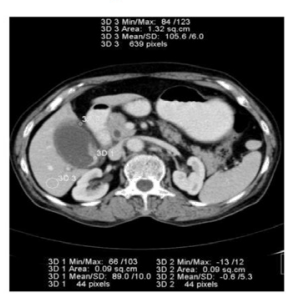

Figure 3E
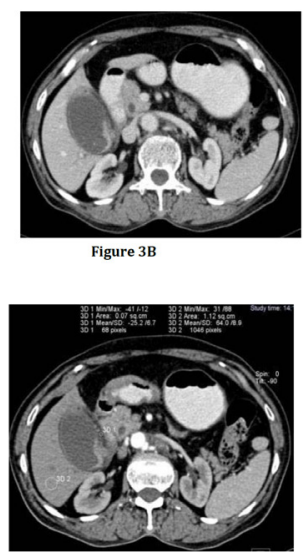

Figure 3D

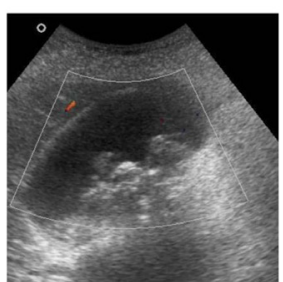

Figure 3F

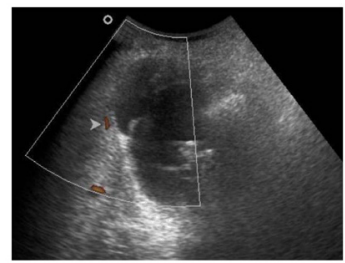

Figure 3G

Figure 3: 76-year old female with diffuse gallbladder wall thickening showing a layered pattern. Arterial phase images (A, C and E) show hyper-attenuation of inner layer compared to the liver parenchyma. The outer wall however showed negative attenuation (E). Venous phase images show hypoattenuation of the gallbladder wall in comparison to liver. Type of pattern was classified as type 3(suggesting malignancy). FNAC was suggestive of benign etiology with possible xanthogranulomatous foci. Color Doppler (F) and power Doppler (G) images showing color flow adjacent to but not within the thickened wall.
Figure 4A

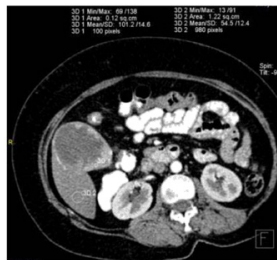

Figure $4 \mathrm{C}$

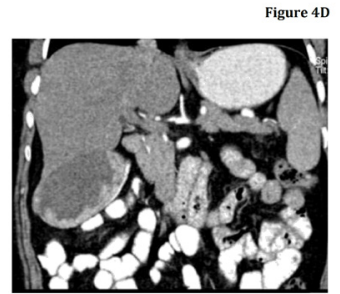

Figure 4E

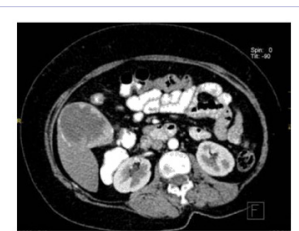

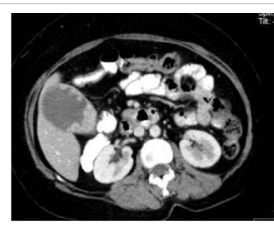

Figure 4B

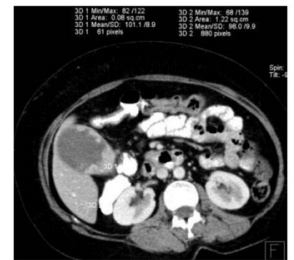

gure 4D Figure 4: 45-year-old female with diffusely thickened gallbladder wall.
Arterial phase images (A, C and E) show hyperattenuation of the wall compared to the liver parenchyma. No layers are seen. Portal venous phase images (B and D) show isoattenuation of the wall compared to the liver parenchyma. The pattern of enhancement was classified as type 3 (suggesting malignancy). However, histopathology was benign.

Among these cases 6 turned out to be carcinoma gallbladder and one turned out to be chronic cholecystitis. Among the remaining 8 cases which did not show a layered pattern of enhancement, seven turned out to be chronic cholecystitis and one turned out to be gallbladder carcinoma. Pearson's Chi-square test showed chi square value of 8.040 with a $p$ value of 0.005 . Thus, in contrast to the previous studies our study results show preferential association of layered pattern of enhancement with the presence of gallbladder carcinoma.

In cases of focal thickening it is difficult to differentiate an area of fibrosis associated with chronic cholecystitis from an area of adenomatous hyperplasia. Our results show diffuse wall thickening in eight patients, three malignant and five benign. Focal wall thickening was seen in seven patients, four malignant and three benign. No statistically significant association was seen between focal gallbladder wall thickening and its malignant nature (Chi-square value of 0.579 and $p$ value of 0.44 ).

The assessment of enhancement in comparison to the liver parenchyma was done in an objective manner by measurement of attenuation within manually drawn ROI. Although the enhancement pattern analysis was done in previous studies, measurement in both phases using regions of interest was not evaluated.

In both arterial and portal venous phases increased 
gallbladder wall attenuation suggested a higher possibility of malignancy. For arbitrary cut off values of $97 \mathrm{HU}$ in portal venous phase and $94 \mathrm{HU}$ in arterial phase, there was a sensitivity of $71 \%$ with a specificity of $87 \%$.

The mean inner wall attenuation values on arterial phase for benign and malignant conditions were $64.5 \mathrm{HU}$ and 94.85 HU respectively. The difference was significant statistically $(2$ tailed t-test, $\mathrm{p}=0.01$ ). The mean inner wall attenuation values on portal venous phase for benign and malignant conditions were $76.12 \mathrm{HU}$ and $99.71 \mathrm{HU}$ respectively. The difference was statistically significant ( 2 tailed t-test, $\mathrm{p}$ value of 0.02 ). These results imply that malignant gallbladder wall thickening shows a higher attenuation value compared to benign gallbladder wall in both arterial as well as portal venous phases. In the study by Yun et al [6], only the subjective comparison of the attenuation of gallbladder wall to that of the liver parenchyma was done. The objective measurement of attenuation value in Hounsfield Units has been done for the first time in our study. The study showed this new finding that the malignant thickened gallbladder wall has a higher attenuation value in arterial and portal venous phases compared to that of benign thickened gallbladder wall.

In the patients with chronic cholecystitis, the most common pattern observed in our study was hypo- attenuation of the wall of gallbladder on portal venous phase. In the study done by Yun et al [6], the most common pattern observed in cholecystitis was iso-attenuation in both the phases. In the carcinoma group, the most common pattern observed in our study was hyperattenuation of gallbladder wall on arterial phase with hypo attenuation in venous phase. The pattern of hyper- attenuation in arterial phase with iso, hyper or hypo-attenuation in portal venous phase was observed in 6 out of 7 cases (86\%). Thus in case of carcinoma gallbladder, our results concur well with the findings of Yun et al [6] which showed that hyper-attenuating inner wall in comparison to liver parenchyma correlates well with malignancy. Our results also partly correlate with the study done by Kim et al [7] which showed that the presence of a thick highly enhancing inner layer (type 2 in their study) corresponded to malignancy. The sensitivity of this finding, according to our study is $86 \%$ with a positive predictive value of $66 \%$. However, in the absence of layered pattern, heterogenous gallbladder wall thickening was classified in their study as type 1 and was correlated with carcinoma or atypical cholecystitis.

In our study, the heterogenous gallbladder wall thickening without layered pattern was mainly seen in chronic cholecystitis and showed iso to hypo attenuating gallbladder wall in comparison to liver parenchyma in 5 out of 8 cases.

In the other three cases, which were false positively identified as malignant based on the criterion of inner wall hyper enhancement; there was lack of layered pattern in two. The third case, although showing a layered pattern of enhancement, showed evidence of a negative attenuation band within the gallbladder wall. This patient had evidence of xanthogranulomatous cholecystitis on cytology.

The presence of increased vascularity within a gallbladder polyp suggests malignancy $[11,12]$. However, the presence of increased vascularity within the gallbladder wall has not been evaluated on power Doppler evaluation to differentiate between malignancy and chronic cholecystitis. In our study, the Doppler examinations of the wall of gallbladder showed visualisation of intramural color flow in four out of eight cases of chronic cholecystitis and in four out of seven cases of malignancy. There was no statistically significant difference seen (Chi-square value 0.07 and $p$ value of 0.78 ).

We consider small number of patients as the main limitation of our study. Further studies with larger number of patients are suggested to confirm our findings.

To conclude, although there is no definite method of distinguishing between gallbladder carcinoma presenting as wall thickening and benign causes of gallbladder wall thickening on imaging, a dual phase MDCT scan may add to the radiologist's confidence in reporting possible cases of malignancy, which helps in appropriate management especially in areas of high incidence of gallbladder carcinoma.

\section{References}

1. Rodriguez-Fernandez A, Gomez-Rio M, Medina-Benitez A, Villar-Del moral J, Ramos-Font C, Ramia-Angel JM, et al. Application of modern imaging methods in the diagnosis of gallbladder cancer. Journal of Surgical Oncol. 2006;93:650-664. DOI: 10.1002/jso.20533

2. Kokudo N, Makuuchi M, Natori T, Sakamoto Y, Yamamoto J, Seki M, et al. Strategies for surgical treatment of gallbladder carcinoma based on information available before resection. Arch Surg. 2003;138(7):741750 .

3. Kalra N, Suri S, Gupta R, Natarajan SK, Wig JD, Joshi K et al. MDCT in the staging of gallbladder carcinoma. AJR. 2006;186(3):758-762.

4. Batra Y, Pal S, Dutta U, Desai P, Garg PK, Makharia G, et al. Gallbladder cancer in India: A dismal picture. J Gastroenterol Hepatol. 2005;20:309-314. doi:10.1111/j.1440-1746.2005.03576.x

5. Smathers RL, Lee JK, Heiken JP. Differentiation of complicated cholecystitis from gallbladder carcinoma by computed tomography. AJR. 1984;143(2):255-259.

6. Yun EJ, Cho SG, Park S, Park SW, Kim WH, Kim HJ et al. Gallbladder carcinoma and chronic cholecystitis: differentiation with two-phase spiral CT. Abdominal imaging. 2004;29(1):102-108. doi:10.1007/ s00261-003-0080-4

7. Kim SJ, Lee JM, Lee JY, Kim SH, Han JK, Choi BI et al. Analysis of Enhancement pattern of Flat Gallbladder Wall Thickening on MDCT to differentiate Gallbladder Cancer from Cholecystitis AJR. 2008;191(3):765-771.

8. Teefy SA, Baron RL, Bigler SA, sonography of the gallbladder: significance of striated thickening of gallbladder wall. AJR. 1991;156(5):945-957.

9. Wibbenmeyer LA, Sharafuddin MJA, Wolverson MK, Heiberg EV, Wade TP, Shields JB. Sonographic diagnosis of unsuspected gallbladder cancer: Imaging findings in comparison with benign gallbladder conditions. AJR. 1995;165(5):1169-1174.

10.Zissin R, Osadchy A, Shapiro-Feinberg M, Gayer G. CT of thickened gallbladder. Br J Radiol, 2003;76(902):137-143.

11. Komatsuda T, Ishida H, Konno K, Hamashima Y, Naganuma H, Sato $\mathrm{M}$ et al. Gallbladder carcinoma: color Doppler sonography. Abdom Imaging. 2000;25(2):194 -\197.

12. Hirooka Y, Naitoh Y, Goto H, Furukawa T, Ito A, Harakawa T. Differential diagnosis of gallbladder masses using color Doppler Ultrasonography. J Gastroenterol Hepatol. 1996;11:840-846. 